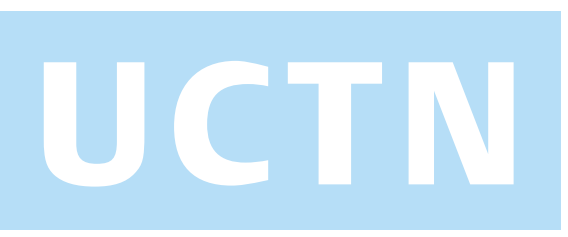

In patients for whom endoscopic sphincterotomy is not possible, the percutaneous transhepatic approach (percutaneous transhepatic biliary drainage; PTBD) is an important alternative. Some patients however do not tolerate longterm PTBD. As a solution to this problem in two patients, we therefore carried out antegrade sphincterotomy (AST) under endoscopic control to improve the position of the papillotome. Both patients presented with cholestasis.

In the first patient, with a BII anastomosis and choledocholithiasis after cholecystectomy, the papilla could only be reached with difficulty. After a small endoscopic papillotomy (EPT), stone extraction failed, even after extracorporeal shockwave li-
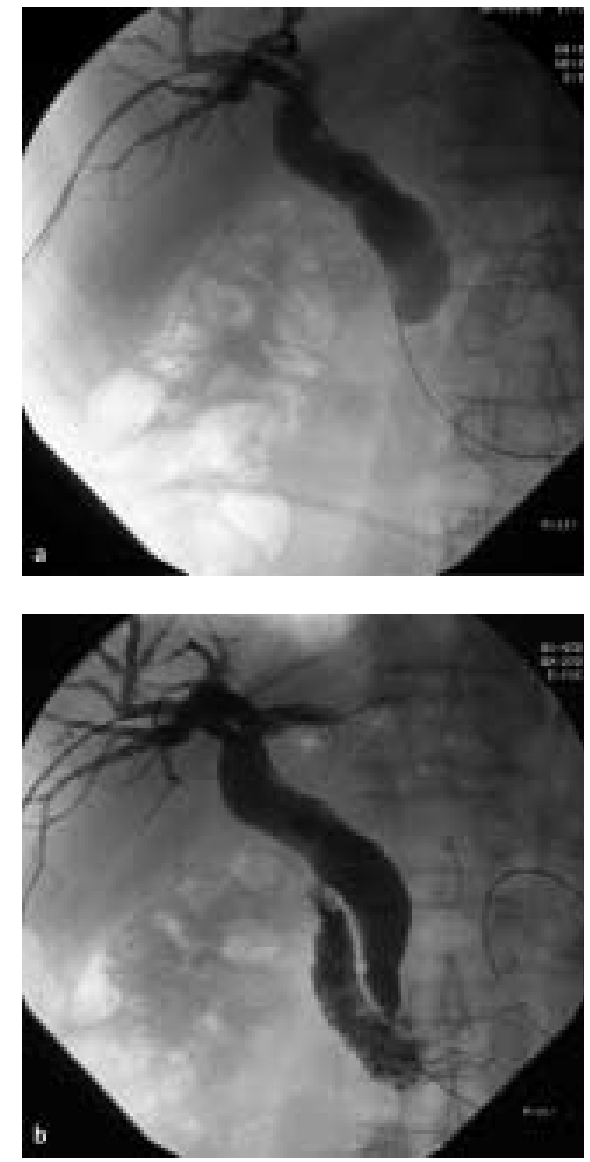

Figure 1 a Before antegrade endoscopic papillotomy (EPT), no spontaneous flow of contrast is seen. b Improved flow is seen after antegrade EPT

\title{
Percutaneous Antegrade Sphincterotomy under Endoscopic Retrograde Control: Report of Two Cases
}

thotripsy. Therefore a successful percutaneous stone extraction was performed followed by insertion of Yamakawa-type PTBD. Extraction of the tube proved to be impossible as there was no spontaneous contrast flow into the jejunum, despite prior EPT and balloon dilation. The second patient, who had a BII anastomosis and an inaccessible papilla, had a benign papillary stenosis which was adequately treated by PTBD. Again, the PTBD tube could not be removed due to the lack of spontaneous flow of contrast (Figure 1 a).

Since both these patients did not tolerate long-term PTBD, we decided to perform an extension of the papillotomy. In the first patient the papilla was again accessible with the endoscope. For the second
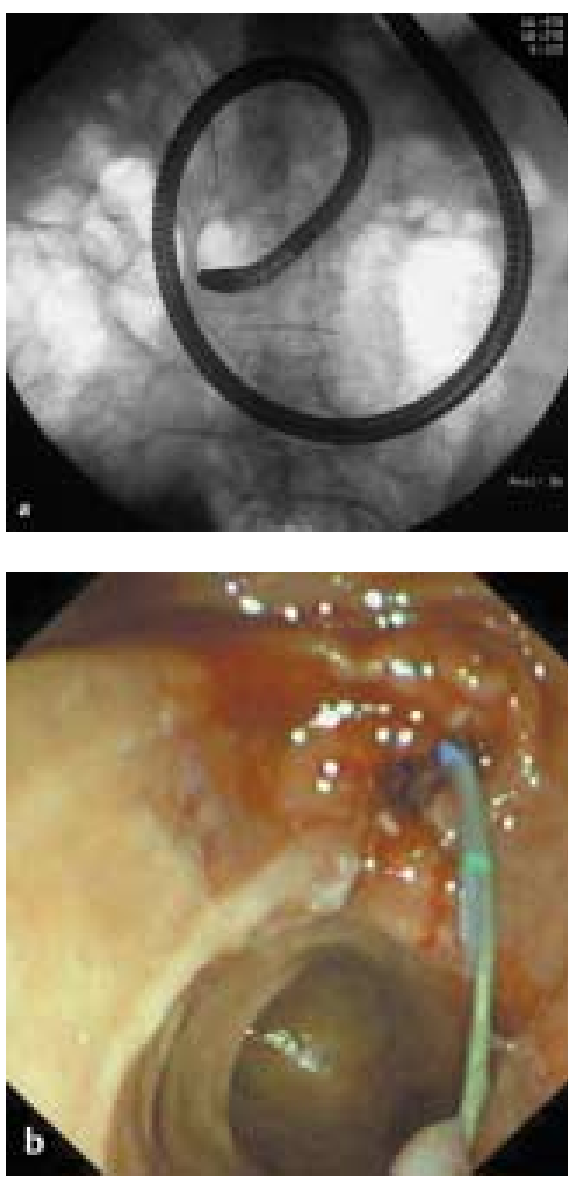

Figure 2 a Fluoroscopy showing antegrade placement of the papillotome under endoscopic control. b Endoscopic control of the antegradely inserted papillotome patient, we inserted a flexible guide wire via the percutaneous tract into the small bowel and, by grasping the distal end of the wire, we were able to pull the endoscope up to the papilla. In both cases, however, the sphincterotome could not be placed properly for a safe cut. We therefore inserted the papillotome over the percutaneous tract, corrected the position with a forceps through the endoscope and performed an extension of the stoma (Figures 2a,2 b). Currently, more than 8 months after extraction of the PTB drain, both patients are free of complaints without any sign of recurrent stenosis (Figure $\mathbf{1 b}$ ).

Particularly in patients whose benign biliary disease has been treated percutaneously, the decision to remove the PTBD tube may be a difficult one to make, since if there is a recurrence a new tract has to be created with all the possible complications this may entail. In our patients, who could not tolerate long-term-PTBD, and in whom percutaneous interventions such as balloon dilation [1] and stenting had failed as well as conventional endoscopic sphincterotomy, we decided to perform the rendezvous maneuver described. AST had already been reported by Wurbs et al. in 1978 [2], in several patients with BII anatomy, in whom the papillotome was introduced via a T-drain and sphincterotomy was done successfully under endoscopic view. Others have reported a similar maneuver during laparoscopic cholecystectomy $[3,4]$. The technique described, which certainly is necessary for only a few patients, represents another step in the development of the so-called rendezvous interventions.

\section{P. Born, W. Sandschin, T. Rösch}

II. Medizinische Klinik,

Klinikum Rechts der Isar, Technische Universität München, Germany 
References

${ }^{1}$ Kawabe T, Komatsu Y, Yamagata M et al. Antegrade guidewire assistant endoscopic papillary balloon dilatation (combined percutaneous and endoscopic technique) for management of common bile duct stone. Gastrointest Endosc 1997; 45: AB 135

${ }^{2}$ Wurbs D, Dammermann R, Ossenberg FW, Classen M. Descending sphincterotomy of the papilla of Vater through the T-drain under endoscopic view. Endoscopy 1978; 10: 199-203

${ }^{3}$ Curet MJ, Josloff RK, Pitcher DE et al. Endoscopic guided antegrade sphincterotomy for complex choledocholithiasis during laparoscopic cholecystectomy. Gastrointest Endosc 1995; 41: 394

${ }^{4}$ Cavina E, Franceschi M, Sidoti F et al. Laparo-endoscopic "rendezvous": a new technique in choledocholithiasis treatment. Hepatogastroenterology 1998; 45: 1430 - 1435
Corresponding Author

\section{P. Born, M.D.}

II. Medizinische Klinik Klinikum Rechts der Isar TU München

Ismaningerstr. 22

81675 München

Germany

Fax: + 49-89-41404905

E-mail: Peter.Born@lrz. tu-muenchen.de 